\title{
LAS HABILIDADES DIRECTIVAS EN LAS INSTITUCIONES DE EDUCACIÓN SUPERIOR. DISEÑO ESTRATÉGICO CON ENFOQUE DE LIDERAZGO.
}

\section{THE MANAGEMENT SKILLS IN THE INSTITUTIONS OF HIGHER EDUCATION. STRATEGIC DESIGN WITH A LEADERSHIP APPROACH.}

\author{
Jimmy Eduardo Ascón Villa, MSc. \\ Máster en Gestión Turística y Licenciado en Turismo (Cuba) \\ Profesor de Dirección y Estrategia en la Facultad de Turismo de La \\ Universidad de La Habana, Cuba. \\ jimmyascon@gmail.com
}

Máryuri García González, PhD.

Doctora en Ciencias de la Educación. (Cuba)

Profesora Titular del Centro de Estudios para el Perfeccionamiento de la Educación Superior

(CEPES), Cuba.

maryuri@cepes.uh.cu

Clara Irene Pedraza Dubernal, MSc.

Máster en Gestión Turística y Licenciada en Estudios Sociocultares.

Docente de la Universidad de La Habana (Cuba).

Asesor en gestión de habilidades directivas en los cuadros y reservas.

clarapedraza@rect.uh.cu

\section{ARTÍCULO DE INVESTIGACIÓN}

Recibido: 26 de enero de 2018

Aceptado: 23 de marzo de 2018

\section{RESUMEN}

Las habilidades directivas cobran cada vez más importancia en las Instituciones de Educación Superior, y como consecuencia en el desarrollo profesional de los dirigentes. Dichas habilidades están estrechamente asociadas a la efectividad del liderazgo. Esta situación motivó la ejecución 
de la presente investigación que tiene como objetivo, evaluar las habilidades directivas de los ejecutivos de la Facultad de Turismo de la Universidad de La Habana, Cuba.

Para alcanzar este propósito, se emplearon métodos empíricos y teóricos, que permitieron realizar un análisis exhaustivo del estado actual en el que se encuentran las habilidades en los dirigentes. Luego de la identificación y caracterización de su situación mediante métodos cualitativos y cuantitativos, se realizó un análisis de los resultados con el uso de la herramienta de la prospectiva Matriz de Impacto Cruzado Multiplicación Aplicada a una Clasificación (MICMAC). Los alcances comprobaron la existencia de un alto nivel de desarrollo de las habilidades y un estilo de liderazgo predominante generando la propuesta de estrategias de mejora y planes de acción para el desarrollo y formación de las habilidades directivas.

Palabras clave: liderazgo, habilidades directivas, prospectiva y estrategias.

\section{ABSTRACT}

Management skills are becoming increasingly important in Higher Education Institutions, and as a consequence in the professional development of leaders. These skills are closely associated with the effectiveness of leadership. This situation motivated the execution of the present investigation, which aims to evaluate the managerial skills of the executives of the Faculty of Tourism of the University of Havana, Cuba.

To achieve this purpose, empirical and theoretical methods were used, which allowed an exhaustive analysis of the current state of the skills in the leaders. After the identification and characterization of their situation by qualitative and quantitative methods, an analysis of the results was made with the use of the prospective tool Matrix of Impact Crossed Multiplication Applied to a Classification (MICMAC). Scopes proved the existence of a high level of skill development and a predominant leadership style, generating the proposal of improvement strategies and action plans for the development and training of managerial skills.

Keywords: leadership, managerial skills, foresight and strategies. 


\section{INTRODUCCIÓN}

Actualmente el análisis de habilidades en un mundo globalizado con un entorno altamente cambiante debe constituir un objetivo primordial de toda actividad, teniendo en cuenta los efectos de diversas variables/factores que han provocado transformaciones en los procesos internos de las universidades, en las estructuras organizativas, los estilos administrativos y las habilidades necesarias para asumir la dirección de personas y recursos. Es necesario que la universidad y sus directivos se preparen en función del cambio, siendo un hecho evidente se debe garantizar en función de los escenarios que se visualizan en el futuro de las universidades.

Es necesario que el mundo cambie, y al ser un hecho evidente se debe garantizar el cambio en función de los escenarios que se visualizan en el futuro de las Instituciones de Educación Superior (IES), en torno a su sostenibilidad y las expectativas del mundo laboral. Tener el dominio del cambio es ante todo una cuestión de voluntad y de capacidad para llevar a todos los trabajadores de la universidad hacia un proyecto común.

El entorno exige una constante renovación en la gestión universitaria, principalmente en las habilidades de sus directivos para que garantice una serie de procedimientos y reglas que mediante la coordinación y organización de los recursos disponibles permita cumplir los objetivos prefijados, en tanto se asumen las tecnologías y el conocimiento.

La prospectiva empresarial es un mensaje de esperanza para todos los que quieren tomar el destino en sus manos. Imaginar un porvenir diferente, es comenzar a cambiar el presente. El sueño hace fecunda la realidad. Para ello se debe dar un salto y un cambio radical en la manera de pensar el futuro y pasar de la anticipación a la acción a través de la apropiación. (Godet, 2016)

Como el futuro cae en el campo de la voluntad, la prospectiva debe tener como objetivo la eficacia de la acción. No hay porqué construir una teoría de la acción, más bien hay que construir una ciencia de la práctica que, antes que una simple aplicación de los métodos científicos a los problemas humanos, constituya un verdadero cambio de perspectiva; el objeto no es observar el futuro a partir del presente, sino observar el presente a partir del futuro. Ese viraje exige decidirse por un futuro entre innumerables posibilidades $y$, por consiguiente, poner en primer lugar, una vez más, el problema de la finalidad de la acción. La reflexión acerca de los fines no se puede separar 
del conocimiento exacto de los posibles medios. La prospectiva permite una confrontación permanente entre los fines, los medios y la realidad de las situaciones presentes (Berger, 1959).

El aumento en la demanda de estudios universitarios, la introducción de tecnologías de la información en el espacio académico y el progresivo acrecimiento de la competencia en todas las actividades relacionadas con el desempeño académico, son hechos que impulsan a los miembros de la comunidad universitaria y de educación superior en general, al aumento de la competitividad de sus instituciones a partir de las habilidades futuras de sus directivos.

El ejercicio del liderazgo por los directivos se justifica por el hecho de que al hablar de centros educativos, se tiene una organización donde intervienen diferentes personas o grupos de éstas, con concepciones, visiones e intereses distintos. Por tanto, esto requiere alcanzar niveles significativos de acuerdos, de visión compartida y de sinergia que promuevan el logro de los fines y objetivos en las Instituciones de Educación Superior (IES).

La nueva universidad está ligada al concepto de progreso y transformación institucional, al diseño de mecanismos adecuados para la toma de decisiones desde las estrategias dictadas en el presente, sin olvidar el liderazgo, pieza angular del desarrollo social y la educación integral característica común en las universidades latinoamericanas, entre las que Cuba distingue como paradigma a seguir. El gobierno ha mantenido una línea estratégica para el constante desarrollo de habilidades en los dirigentes, a partir de métodos y formas en la política de cuadros para la construcción de los escenarios futuros, la cual se ha precisado, favorecido y mejorado, en correspondencia a los contextos de cada momento histórico del proceso revolucionario.

En estudios realizados por los integrantes del proyecto institucional de Gestión Prospectiva de Habilidades Directivas en la Universidad de La Habana, y su aplicación en la Facultad de Turismo se observa que no existen directrices estratégicas que normen y exijan un cambio en la manera de conocer y proyectar las habilidades directivas en la práctica habitual. Se hace indiscutible que los directivos en este contexto deben enfocarse en mejorar el proceso de toma de decisiones a partir del desarrollo de habilidades que les permitan superar las dificultades de índole administrativo a lo largo de los eslabones de la cadena de mando. Tales dificultades pueden ser producto de la carencia de estrategias de gestión con empleo de la prospectiva para determinar las pautas a seguir en el desarrollo de habilidades en los dirigentes, asociadas al carácter limitado del liderazgo, 
y la falta de una perspectiva sistémica de la realidad en el empleo de técnicas para la construcción de futuros.

\section{REVISIÓN TEÓRICA}

\section{Las habilidades directivas en las Instituciones de Educación Superior}

Según el Diccionario Larousse: "habilidad" es la "capacidad para hacer una cosa". Son sinónimos: destreza, capacidad, arte, competencia, aptitud, maña, entre otros. Supone, además de conocimientos sobre una cosa (conceptos, procesos, técnicas, sus interrelaciones con "otras cosas"), la posibilidad de aplicar las mismas de manera efectiva, en las circunstancias que resulte necesario y su conversión en métodos de trabajo y de comportamiento habituales (Codina, 2012).

Knapp (2007) señala con relación al término habilidad directiva que es la capacidad del individuo, adquirida por el aprendizaje, capaz de producir resultados previstos con el máximo de certeza, con el mínimo de tiempo y economía y con el máximo de seguridad.

Según Madrigal (2009) la adquisición y aprendizaje de las habilidades directivas tiene como propósito producir resultados previstos con máxima certeza. Estas habilidades se enlazan a una tarea, implican un entorno, se manifiestan en la realización de las labores con regularidad y eficacia, y, sobre todo, se aprenden.

Las habilidades directivas son el vehículo mediante el cual la estrategia y la práctica de la administración, las herramientas y las técnicas, los atributos de la personalidad y el estilo trabajan para producir resultados eficaces dentro de las organizaciones. En otras palabras, las habilidades directivas son los bloques de construcción sobre los que descansa la administración efectiva (Whetten \& Cameron, 2011).

Las habilidades directivas son el conjunto de capacidades y conocimientos que una persona necesita para realizar las actividades de administración, de liderazgo y de coordinación en el rol de gerente de una determinada organización. (Cristancho \& Rojas, 2012)

La organización representa el contexto donde el directivo despliega y aplica sus conocimientos para generar beneficios. La rapidez con que se trasforma el entorno organizacional, el creciente 
desarrollo tecnológico, la competencia, el comportamiento ascendente de la exigencia de los clientes, demandan del gerente un comportamiento y unas habilidades directivas para llevar a cabo estrategias, programas y estructuras que permitan a la organización la anticipación a los cambios que pueden afectarle, y que a su vez sea sostenible en el tiempo. (Cristancho \& Rojas, 2012) El perfeccionamiento de las habilidades directivas básicas para la gestión constituye el mayor desafío en el acontecer empresarial.

Para un mejor entendimiento del término "habilidades directivas", Whetten y Cameron (2011) establecen cinco propiedades que las particularizan:

Primeramente, son conductuales; no son atributos de la personalidad o tendencias de estilo. Las habilidades directivas consisten en conjuntos identificables de acciones que los individuos llevan a cabo y que conducen a ciertos resultados. Las habilidades pueden ser observadas por otros, a diferencia de los atributos que son puramente mentales o fijos en la personalidad. No obstante, en tanto que los individuos con diferentes estilos y personalidades aplican sus habilidades de manera diferente, existe un grupo central de atributos en el desempeño eficaz que son comunes a través de toda una gama de diferencias individuales.

En segundo lugar, las habilidades administrativas son controlables. El desempeño de estos comportamientos se encuentra bajo control del individuo, el cual las puede demostrar, practicar, mejorar o limitar. Es cierto que las habilidades pueden implicar a otras personas y que requieren de trabajo cognoscitivo, pero son comportamientos que la gente puede controlar por sí misma.

En tercer lugar, pueden ser desarrolladas. Es posible mejorar el desempeño. A diferencia del cociente intelectual (ci) o de ciertos atributos de la personalidad o del temperamento, los cuales permanecen relativamente constantes a lo largo de la vida, los individuos pueden mejorar su competencia en el desempeño de habilidades por medio de la práctica y la retroalimentación. Los individuos pueden progresar de menor a mayor competencia en las habilidades directivas.

En cuarto lugar, se puede considerar que están interrelacionadas y se traslapan. Es difícil demostrar una sola habilidad aislada de las demás. Las habilidades no son comportamientos simplistas y repetitivos, sino conjuntos integrados de respuestas complejas. Los directivos eficaces desarrollan un conjunto de habilidades que se superponen y se apoyan unas a otras, y que permiten flexibilidad en el manejo de situaciones diversas. 
En quinto lugar, las habilidades directivas a veces son contradictorias y paradójicas. Por ejemplo, no todas las habilidades directivas fundamentales tienen una orientación suave y humanista, ni todas son impulsoras y marcan una dirección. No están orientadas exclusivamente hacia el trabajo en equipo o hacia las relaciones interpersonales, ni exclusivamente hacia el individualismo o el carácter técnico. Los directivos más eficaces suelen poseer una variedad de habilidades, y algunas de ellas parecen incompatibles.

Los directivos más eficaces son tanto participativos como muy impulsores, tanto alentadores como competitivos. También deben poseer la capacidad de ser flexibles y creativos, y al mismo tiempo controlados, estables y racionales (Whetten \& Cameron, 2011).

\section{Las destrezas como habilidades directivas}

Para Katz (1974), citado por Codina (2001), establece como habilidades directivas básicas indispensables la posesión de destrezas técnicas, conceptuales y humanas:

$\checkmark$ La destreza técnica, como capacidad de utilizar las herramientas, procedimientos y técnicas de una disciplina especializada, como necesitan el ingeniero o el médico, para efectuar lo que llama "mecánica de su trabajo".

$\checkmark$ La destreza humana, como capacidad de trabajar con otras personas, como individuos o como grupos, y de entenderlos y motivarlos.

$\checkmark$ La destreza conceptual, como capacidad mental de coordinar e integrar todos los intereses de la organización como un todo.

Según Sutevski (2009), fundador y director ejecutivo de Sutevski Consulting y creador de excelencia empresarial a través del pensamiento innovador:

Las destrezas técnicas son los conocimientos y capacidades indispensables que necesita el gerente para desempeñar correctamente las funciones inherentes a su cargo. Las habilidades técnicas son más importantes para los gerentes de primer nivel; en una jerarquía de abajo hacia arriba, las destrezas técnicas van perdiendo su importancia. 
Destrezas conceptuales: Conocimiento o habilidad de un gerente para un conocimiento más abstracto, lo que significa que puede ver fácilmente el todo a través del análisis y diagnóstico de diferentes estados; de esta manera pueden predecir el futuro del negocio. Este tipo de destrezas son vitales para los altos directivos, a medida que se pasa de la parte inferior de la jerarquía gerencial a la cima, su importancia aumentará.

Destrezas humanas: Conocimiento y habilidad de un gerente para trabajar con la gente. Una de las tareas de gestión más importantes es trabajar con personas; estas destrezas permiten a los gerentes convertirse en líderes y motivar a los empleados para lograr mejores resultados, además les ayudará a hacer un uso más eficaz del potencial humano en la empresa. Se consideran las habilidades más importantes para los directivos y son requeridas en todos los niveles jerárquicos de la empresa (Sutevski, 2009).

\section{El liderazgo y sus estilos}

El origen de la palabra "liderar", viene del vocablo "laed", común a todas las antiguas lenguas del norte de Europa (holandés, alemán, anglosajona, noruega, danesa, sueca), y significa senda, ruta, curso de un barco en el mar, trayecto. Su esencia está ligada a la palabra líder que según Menguzzato y Renau (1993) es "la persona a quien le es posible influir en las actitudes y opiniones de los miembros de un colectivo".

Desde tiempos inmemorables el liderazgo se ha evidenciado en las sociedades humanas a través de los grandes emperadores, reyes, guerreros, empresarios, gestores y directivos entre otros, los cuales gracias a sus logros, estamparon sus nombres en la historia de la humanidad, siendo en muchos casos los sujetos de estudio para diferentes temas; en este caso en especial, el liderazgo. (Ryo, 2015)

En un principio el estudio del liderazgo se basó en definir estructuras sobre cómo se podría dirigir a la sociedad y en el análisis de los grandes líderes de la historia del mundo, identificando cuáles eran esas características que los diferenciaban de los demás, llegando a la gran conclusión que los líderes nacían, no se hacían. Es entonces, en el siglo XX cuando esta hipótesis comienza a ser refutada por los nuevos especialistas en el tema, ya que todos los tipos de liderazgo que se presentan hoy en día, pueden ser aprendidos. Se trata de habilidades de dirección, de influir sobre las personas para conducirlos al logro de metas y objetivos compartidos. 
Las definiciones dadas al liderazgo por teóricos e investigadores entre 1900 y 1989, permiten obtener una visión de la evolución conceptual de este fenómeno. Entre 1900 y 1929 el liderazgo es definido a partir de las características de control y poder centralizado. En la década de los 30 se define como la interacción entre los rasgos específicos de una persona y de otros muchos. En los años 40 se considera como el resultado de las habilidades de persuadir de manera directa, como la fuerza principal que estimula, motiva y coordina la organización en la consecución de los objetivos. En los 50 es visto como una relación en la que se desarrollan metas compartidas, la solución de problemas y la aceptación de la autoridad plena ejercida por el líder. El aporte más importante de esta década fue la influencia de las ideologías democráticas en la definición de este concepto.

A partir de 1960 existe una unicidad en la comprensión del concepto como una conducta que influye sobre las personas para compartir metas, pero dicha influencia es unidireccional. En esta época los términos líder y liderazgo se usan indistintamente como sinónimos, igualmente se confunde con la dirección. El período de los 70 es notorio la falta de coherencia de los autores para definir el liderazgo, aunque se desarrolló una teoría en la que insistía que este fenómeno debía distinguirse del concepto de autoridad. Otro valioso aporte de esta época es el hecho por James Burns en 1978 con la teoría sobre el liderazgo transaccional. (Chamorro, 2005)

Iniciado el sigo XXI, el concepto de liderazgo se une de manera inseparable al cambio y a la transformación. La visión del liderazgo en un valor sustancial en las habilidades directivas de poder ejercer como motor del cambio, ya sea social, económico o de otro tipo. Ha surgido el líder multidisciplinar. Un liderazgo de múltiples vértices y de enormes capacidades creativas. (Bayón, 2015)

Las diferentes definiciones del término liderazgo, no tienen problemas en sí mismas, la mayoría dicen fundamentalmente lo mismo: el liderazgo tiene que ver con una persona que logra que otras hagan. Donde las definiciones difieren es en cómo los líderes motivan a sus seguidores, y en quién tiene voz y voto para diseñar los objetivos del grupo o de la organización. (Marco, 2000)

Lo curioso de todo lo expuesto es la velocidad con la que ha evolucionado el concepto después de que permaneciera dormido durante tantos años. 
Según Chamorro (2005) el empresario Ralph M. Stogdill formuló una definición clásica de liderazgo: "el proceso de influir sobre las actividades de un grupo organizado en sus esfuerzos hacia el establecimiento y logro de metas". Gómez (2008) expresó que James McGregor Burns planteó: "El liderazgo sobre los seres humanos se ejerce cuando un grupo de personas con ciertos motivos y fines se moviliza, en competencia o en conflicto con otros recursos institucionales, políticos, psicológicos, etc., para inducir, comprometer y satisfacer los motivos de los seguidores".

Chamorro (2005) plantea que los importantes investigadores Warren Bennis y Burton Nanus lo describen como: "[...] la fuerza central que se encuentra detrás de las organizaciones de éxito y que ayuda a las organizaciones a desarrollar una nueva visión de lo que puede ser, y luego las moviliza para el cambio hacia la nueva visión [...] un factor esencial del liderazgo es la capacidad para influir y organizar el significado para los miembros de la organización".

El autor de la presente investigación se adscribe a la definición de liderazgo como: "clase de influencia mediante la que se puede lograr que los miembros de una organización colaboren voluntariamente y con entusiasmo en el logro de los objetivos organizacionales" (Menguzzato \& Renau, 1993). Por décadas el liderazgo ha presentado varios enfoques o teorías las cuales han sido causa de numerosos estudios. En la teoría como en la práctica, muchos han tratado de definir lo que diferencia a un verdadero líder de las masas. (Leadership-Central, 2016).

Las diferentes teorías se pueden agrupar por era o períodos de tiempo, destacando la era de los "Rasgos" desde 1800 a 1940, donde se incluye la Teoría del Gran Hombre, la era de las Teorías "Behavioristas" de 1940 a 1960, la era de la "Contingencia" donde se destacan grandes autores como Paul Hersey, Kenneth Blanchard y Fred Fiedler. La era del "Nuevo liderazgo" desde 1980 a1990 donde se encuentran la Teoría Transaccional y la Transformacional. Por último, la era "Posttransformacional" desde el año 2000 en adelante. (Ryo, 2015)

\section{Estilos de liderazgo:}

Se puede apreciar en los equipos y en las organizaciones diferentes estilos de liderazgo como:

Líderes Transaccionales: Identifican qué necesitan sus subordinados para cumplir sus objetivos, aclaran funciones y tareas, instauran una estructura organizacional, premian el desempeño y toman en cuenta las necesidades sociales de sus seguidores. Trabajan intensamente e intentan dirigir a la organización con toda eficiencia y eficacia. 
Liderazgo Transformacional: prestan mayor atención a sus seguidores en cuanto a sus necesidades e intereses de desarrollo ayudándolos a ver los viejos problemas de nuevas formas, son capaces de lograr un esfuerzo extra para lograr las metas del grupo. Los líderes transformadores hacen partícipe a los subordinados de las decisiones que se toman en la organización. Se caracterizan por su carisma proporcionando visión y sentido de misión, originan orgullo, obtiene respeto y confianza, son inspiradores y comunican altas expectativas, estimulan intelectualmente promoviendo la inteligencia y la solución cuidadosa de problemas e instruyen y aconsejan a sus subordinados.

Liderazgo Carismático: tienen un alto poder de referencia, que es el deseo del influenciado de parecerse o identificarse con el influyente y que parte de él, de la necesidad de influir en los demás, de tener gran seguridad en sí mismos, dominio y convicción o la capacidad de persuadir a sus seguidores de que la tienen.

Liderazgo Autocrático o Líder Centrado en las Tareas: suprema preocupación por la tarea misma y no por los trabajadores como personas. Se caracteriza por tomar decisiones de un modo unilateral y limita la participación de los subordinados en la definición de los objetivos y demás actividades, centraliza su autoridad, es dogmático y espera obediencia, su poder resulta de su capacidad de otorgar recompensas o castigos. Se correlaciona positivamente con la productividad. Tiende a reducir la satisfacción y la cohesión.

Liderazgo Democrático o Líder Centrado en las Personas: consulta a sus subordinados respecto de acciones y decisiones probables y alienta su participación. No está consistentemente relacionado con la productividad. Se establece una comunicación bilateral entre el líder y el equipo. El grupo recibe una mayor información sobre los problemas que le atañen. El liderazgo orientado a las personas tiende a aumentar la satisfacción de ellas; también tiende a aumentar la cohesión del grupo.

Liderazgo Liberal o "laissez-faire": el grupo tiene libertad completa para la toma de decisiones, con mínima participación en debate. El líder se limita sólo a hacer comentarios sobre las actividades del grupo. En este caso el líder no aparece como guía, afectando así la producción, con un bajísimo rendimiento por parte del personal. 
Líder Ausente: No toma decisiones, sino que las demora. Proyectos en los que no hace ni dice nada. Se concentra en sus propias metas. Da el poder pero no lo delega. No motiva, no inspira.

Líder Sin Autoridad: normalmente está determinado por personas nuevas en la empresa, que carecen de conocimiento de los miembros y autoridad formal, y conocen los problemas existentes, lo que constituye una oportunidad de mejora.

Seudoliderazgo: realizan muchos discursos con mucha pasión, y aparentan estar muy comprometidos con el liderazgo y las personas. Se orientan más hacia el manejo de la imagen. No se comprometen de manera humilde y presumen de sus estudios. Son inseguros, egocéntricos y manipuladores, buscan el poder para beneficio personal.

\section{Conceptos de la prospectiva estratégica.}

El término estrategia es adquirido del terreno militar y es llevado al área económica debido a su esencia, la cual es competir y actuar frente a un enemigo para alcanzar el objetivo que se ha propuesto. La Dirección Estratégica es definida por Ronda y Marcané (2004) como: "el proceso interactivo y holístico de formulación, implantación, ejecución y control de un conjunto de estrategias, que garantiza una interacción proactiva de la organización con su entorno, para contribuir a la eficiencia y eficacia en el cumplimiento de su objeto social". La estrategia es el modelo anticipado de comportamiento, que recoge elementos positivos y diferentes del pasado, un conjunto de conocimientos acumulados de la organización y del entorno que deben ser ordenados, integrados y organizados de forma tal que permita identificar a qué renunciar si se compromete el futuro, optimizar la posición y los resultados de la instalación a corto, medio y largo plazo aprovechando sus ventajas competitivas y las debilidades de la competencia (Guerra, 2009).

Para Ascón (2017) la estrategia se corresponde con un conjunto de líneas, directrices o caminos creativos a seguir en la empresa a partir de las condiciones internas, los elementos del entorno y los factores que determinan el éxito empresarial en general, y que engloban un conjunto de actividades coordinadas que permiten el logro de los objetivos propuestos desde cada unidad empresarial de negocio. Hay autores que consideran a la prospectiva como ciencia (autores de la actualidad, pero desde los inicios del concepto, Berger lo definió como ciencia), pero aún está en proceso y en polémica este tema. Hay otros que tienen la tentación de declararla disciplina, pero más bien, al decir de Godet (2012), es la indisciplina. Baena subraya que dados los planteamientos de la ciencia dominante, no se puede considerar como tal si no tiene referencia empírica en la cual se comprueben los hechos. Otros plantean que no puede existir como ciencia ya que no tiene 
objeto de estudio, porque el futuro no existe y por definición deja de serlo: en el momento en que se concreta ya es presente, no es futuro.

La prospectiva cambia la manera de pensar y la engrandece, basado en las tendencias, experiencias o presentimientos, por tanto, enriquece la existencia. A continuación, se presentarán algunas definiciones de prospectiva en las cuales se puede apreciar una evolución de las mismas: El concepto fue acuñado a comienzos de los años 50 por el pensador francés Gaston Berger que lo definía como "la ciencia que estudia el futuro para comprenderlo y poder influir en él". Berger añadía y recalcaba que mediante la prospectiva se debía "observar lejos, ampliamente y profundamente, pensar en el hombre y asumir riesgos" (Astigarraga, 2016). A partir de este análisis se argumentan los principales métodos y técnicas procedimentales de la prospectiva. De acuerdo con Miklos \& Tello (2007) la prospectiva es creatividad, análisis y capacidad de integrar deseos y futuros esperados con las condiciones actuales.

El Instituto de Prospectiva Estratégica, en 1999, definió la prospectiva como una disciplina con visión global, sistémica, dinámica y abierta que explica los posibles futuros, no sólo por los datos del pasado sino fundamentalmente teniendo en cuenta las evoluciones futuras de las variables (cuantitativas y, sobre todo, cualitativas) así como los comportamientos de los actores implicados, de manera que reduce la incertidumbre, ilumina la acción presente y aporta mecanismos que conducen al futuro aceptable, conveniente o deseado. Según Serra (2004) es una disciplina científica con la aspiración a funcionar según los principios fundamentales de la ciencia, a saber: publicidad y repetitividad. Antes había señalado que la prospectiva era la ciencia que estudia el futuro para comprenderlo y poderlo influir.

Concluyendo y no menos importante es bueno precisar dos conceptos anglosajones de manejo con respeto a la prospectiva, estos son:

$\checkmark$ Forecast: textualmente previsión, mirar hacia delante; sin embargo, en contraparte, se creó el concepto foresight que también quiere decir previsión.

$\checkmark$ Foresight: concepto que se ha popularizado mucho en Europa; se presenta como una actualización o superación de la noción de prospectiva. 


\section{MATERIALES Y MÉTODOS}

La presente investigación es "descriptiva", porque se caracterizan las habilidades directivas como objeto de estudio. Además, se refleja un análisis "cuantitativo" y "cualitativo". De la misma manera, según la estrategia metodológica abordada, se puede clasificar como "investigación-acción", porque se parte de la realidad existente en los directivos y reservas para diagnosticar su situación.

\section{Fundamentación teórica de la investigación}

Se recurrió a la revisión de artículos científicos, trabajos de diplomas, tesis de maestría, para encontrar la información bibliográfica que respalda teórica y conceptualmente la temática en estudio.

\section{Métodos teóricos:}

> Histórico-Lógico: El método histórico permite el estudio de la evolución de las habilidades directivas, así como las herramientas de la gestión prospectiva y estratégica, sus tendencias actuales y la posibilidad de aplicación en la Facultad de Turismo de la Universidad de La Habana. Se analizarán investigaciones realizadas por importantes exponentes de la visión de futuro en el campo de las Instituciones de Educación Superior.

> Hipotético-deductivo: El uso de este método facilita la formulación de la hipótesis de la investigación como relación entre las variables habilidades directivas en los dirigentes y sus estrategias de mejora en función del liderazgo futuro. El planteamiento de esta hipótesis y el desarrollo teórico de la investigación permitirá arribar a nuevas conclusiones.

> Inductivo- Deductivo: Permite identificar las tendencias más recientes en cuanto a la gestión de habilidades directivas en las IES a nivel internacional y las posibilidades de aplicarlas a la realidad de la Facultad de Turismo de La Universidad de La Habana.

> Análisis-síntesis: Posibilita la búsqueda y el procesamiento de la información a partir de la bibliografía consultada y del estudio teórico de la temática investigada. Permite determinar las características del estudio de escenarios futuros y las relaciones entre la gestión de los principales actores y las variables determinadas para la mejora de las habilidades directivas de los dirigentes en el presente.

\section{Caracterización de la Universidad de la Habana y la Facultad de Turismo}

Se caracterizó la Universidad de La Habana y la Facultad de Turismo. Para ello, se recurrió a los 
métodos teóricos enunciados en la etapa anterior. Además, se realizó el análisis documental, para lo cual se consultó el Manual de Procedimientos de la Universidad de La Habana y de la Facultad de Turismo, y sus registros con respecto al trabajo con los directivos. Para la investigación y análisis del liderazgo en los directivos de la facultad se hizo necesaria la caracterización del orden jerárquico que presentan.

\section{Evaluación de las habilidades directivas en los directivos de la Facultad de Turismo}

La observación, entrevista y encuesta fueron los métodos empíricos de investigación utilizados para evaluar la situación actual de las habilidades directivas.

Observación: Se aplicó con el propósito de analizar las habilidades.

\section{- Encuestas:}

Se aplicaron para conocer el estado actual de las habilidades, según la percepción de su realización e importancia. Se empleó el método intencional encuestando a los directivos de la facultad.

\section{Técnicas Empleadas en el análisis de resultados}

\section{Matriz de Impacto Cruzado Multiplicación Aplicada a una Clasificación:}

El análisis estructural es una herramienta de estructuración de una reflexión colectiva; ofrece la posibilidad de describir un sistema con ayuda de una matriz que relaciona todos sus elementos constitutivos. Partiendo de esta revisión, este método tiene por objetivo, hacer aparecer la revisión de variables influyentes y dependientes y por ello las variables esenciales a la evolución del sistema: "Plantear las buenas preguntas e identificar las variables clave"

\section{ANÁLISIS DE RESULTADOS}

\section{Resultados de la encuesta aplicada a los directivos de la Facultad de Turismo}

La información obtenida a partir de las encuestas aplicadas a los directivos de la Facultad de Turismo de La Universidad de La Habana ofrece datos significativos en cuanto a la incidencia de otros aspectos instructivos en el desarrollo de las habilidades directivas. De los directivos 
encuestados el 43\% poseía una experiencia menor a los 10 años, el $48 \%$ entre 10 y 15 años y solo el $9 \%$ entre 15 y 20 años. Se observa un ligero equilibrio en cuanto a sexo, ya que el $57 \%$ fueron mujeres, mientras que el $43 \%$ pertenece al sexo masculino. A su vez están bastante equiparadas las muestras con respecto a la edad, puesto que el $43 \%$ oscila entre los 28 y 35 años, el $48 \%$ posee entre 35 y 50 años, mientras que solo el $9 \%$ tiene entre 50 y 60 años de edad.

Como resultado de la encuesta aplicada se reveló que, según las percepciones de los cuadros de las facultades objeto de estudio, las habilidades de mayor importancia, según el nivel de frecuencia en la selección son: las destrezas humanas (74\%); el compromiso organizacional (65\%), visión de futuro (78\%), capacidad de búsqueda de información (70\%) y tolerancia a la presión (69\%), como parte de las habilidades personales; la dirección y participación en reuniones y el trabajo en equipo, ambas (78\%), el hablar en público (91\%) y la gestión de reuniones (70\%), todas ellas como habilidades interpersonales; destacaron a su vez, las habilidades grupales o de gestión como las de mayor relevancia, encontrándose aquí el liderazgo con un 100\%, la gestión financiera (89\%), la innovación (87\%), y la planificación y organización (83\%). A la par, se determinaron, según resultados de selección de los directivos, que entre las habilidades que presentan un bajo nivel de importancia se encuentran, la polivalencia (69\%) y la sensibilidad organizacional (78\%), como habilidades personales; la asertividad (87\%) y la escuela activa (100\%), todas ellas como habilidades interpersonales; la gestión de relaciones con los stakeholders (74\%) y la gestión sociocultural de equipos (87\%).

Todo ello condicionado, en mayor medida, por el desconocimiento de los encuestados en relación a las definiciones de las habilidades directivas analizadas, es decir, según la interpretación realizada de cada término, le otorgaban una puntuación, generando esto, que en reiteradas ocasiones, habilidades muy importantes en el desempeño de la gestión administrativa de las universidades como por ejemplo la formulación estratégica o la gestión del ciclo directivo no resultaran relevantes en el análisis. Lo que evidencia, además, que es necesario trabajar en el desarrollo de acciones y actividades encaminadas al fortalecimiento de este aspecto, puesto que si los cuadros no conocen las definiciones correctas de cada una de las habilidades directivas difícilmente podrán desempeñarlas.

El criterio de los encuestados en relación a los motivos que influyen negativamente en la mejora de habilidades directivas se expresa en la figura 2. Dichos criterios evidencian que la falta de tiempo entre los cuadros para la formación de sus habilidades constituye el factor de mayor incidencia 
(96\%), seguido de la ausencia de programas de capacitación destinados al desarrollo de las mismas (78\%).

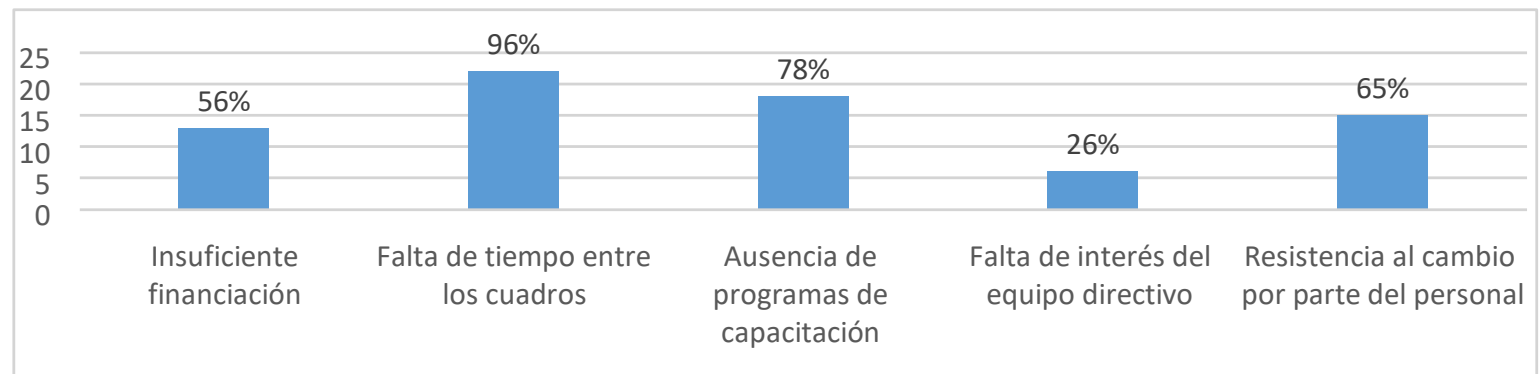

Figura 1.. Respuestas de los directivos para la variable "influencias negativas".

Fuente: Elaboración propia

Hechos que deben ser evaluados con objetividad, puesto que constituyen un problema evidente para la formación de habilidades directivas en los cuadros, lo que incide negativamente en la gestión administrativa del sector universitario.

Al evaluar las temáticas de mayor y menor incidencia en el desempeño de las habilidades directivas destacaron como temáticas más influyentes la planificación estratégica, las herramientas de gestión prospectiva estratégica, la gestión del proceso enseñanza-aprendizaje, la gestión de personas y la responsabilidad social universitaria; así como otras de menor influencia como la comercialización, el diseño organizativo, el desarrollo de infraestructuras, el multiculturalismo y la gestión de la innovación.

Al analizar la importancia atribuida por los cuadros encuestados a las actividades de formación de habilidades directivas, como muestra la Figura 2, se determinó que dentro de las más relevantes se encuentran los cursos de formación presencial (87\%), las consultorías (52\%), los entrenamientos y talleres (ambas 40\%) de frecuencia de selección; a la par que se revelaron como menos importantes el benchmarking (61\%), y de importancia media los casos de estudio y los cursos de formación online, ambas (43\%). 


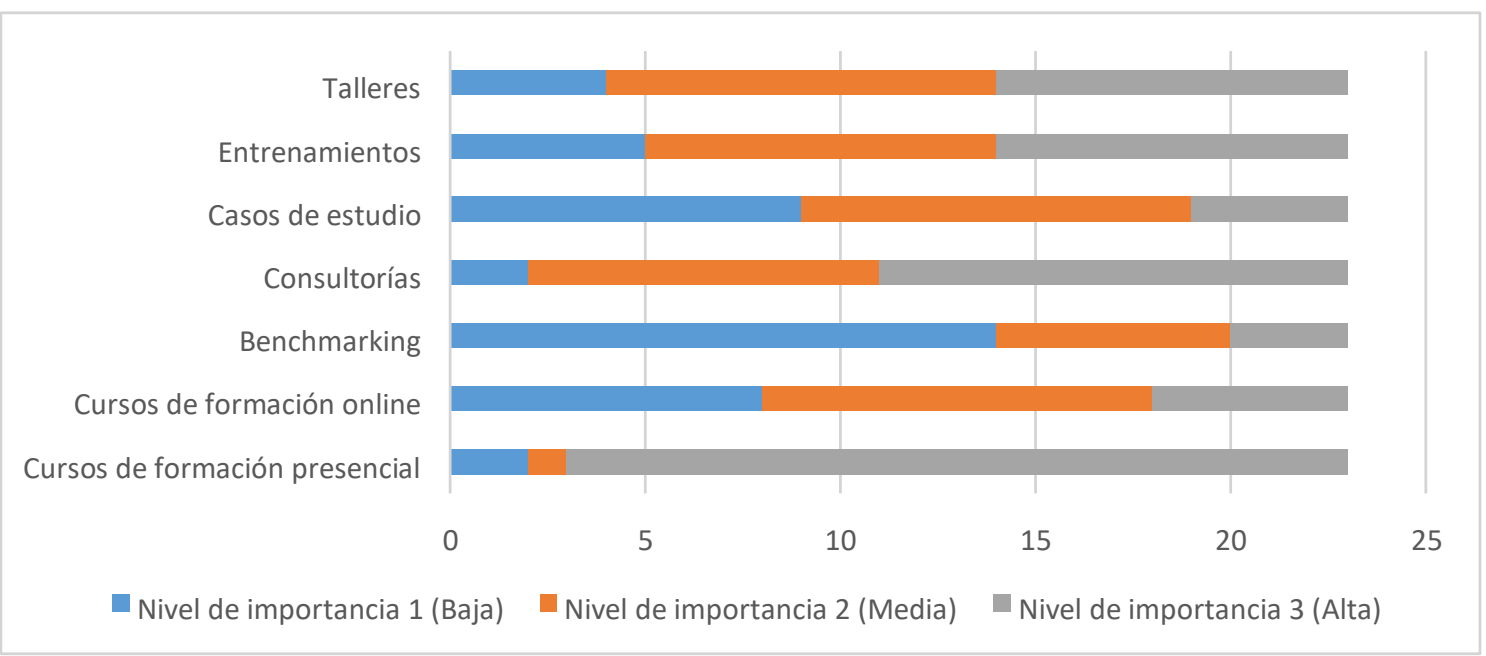

Figura 2. Análisis de la variable "actividades".

Fuente: Elaboración propia

Estos resultados están condicionados, en parte, por el desconocimiento de términos como Benchmarking por una parte de los directivos de la Facultad de Turismo, que no le atribuyen a esta actividad la importancia que realmente posee. A su vez, otra limitación radica en las condiciones tecnológicas para el servicio de acceso a Internet, lo que dificulta el empleo de cursos de formación online.

Finalmente, en el análisis de la encuesta, al brindar a los directivos la oportunidad de desarrollar las decisiones más significativas que han tenido que enfrentar a lo largo de su experiencia, se detectó como una limitación la disponibilidad del tiempo, ya que la mayoría de los espacios para responder las preguntas de esta índole no fueron completados. De ahí la necesidad de realizar entrevistas para contrastar informaciones imprecisas.

Tabla 1. Resultados de la pregunta 5 de la encuesta habilidades directivas.

\begin{tabular}{|l|l|}
\hline Decisiones difíciles o significativas & Habilidad para su solución \\
\hline Designar o proponer personas a cargos & Desarrollo de funciones \\
\hline Mediar conflictos & Negociación, diálogo, persuasión \\
\hline $\begin{array}{l}\text { Sancionar a jóvenes talentosos por errores } \\
\text { graves }\end{array}$ & Tolerancia a la presión \\
\hline Negar la entrada a la Universidad & Compromiso social y responsabilidad \\
\hline
\end{tabular}




\begin{tabular}{|l|l|}
\hline Reclamar derechos personales & Confianza propia \\
\hline
\end{tabular}

Fuente: Elaboración propia

Según la codificación que se empleó para la interpretación de los resultados, en la encuesta dirigida a los cuadros, menos de 16 puntos se caracteriza como Seudoliderazgo, entre 17 y 30 puntos como un líder Autocrático, entre 31 y 40 puntos como un líder transaccional y más de 40 puntos como un líder transformacional. En la Facultad existe un predominio de líderes transformacionales ocupando un total de $70 \%$ en las encuestas, se caracterizan por preocuparse por sus subordinados y compañeros ayudándolos a superar problemas de diferentes índoles, se esfuerzan el doble para lograr las metas del grupo. Ellos hacen partícipe a los subordinados de las decisiones que se toman en la facultad. Por lo general son muy carismáticos, logrando obtener respeto, confianza, e inspiran y comunican altas expectativas. Con el $22 \%$ se sitúan los líderes transaccionales que trabajan intensamente e intentan dirigir a la organización con toda eficiencia y eficacia. Solo el 8\% están los líderes autocráticos que tienen una suprema preocupación por la tarea misma y no por los trabajadores como personas.

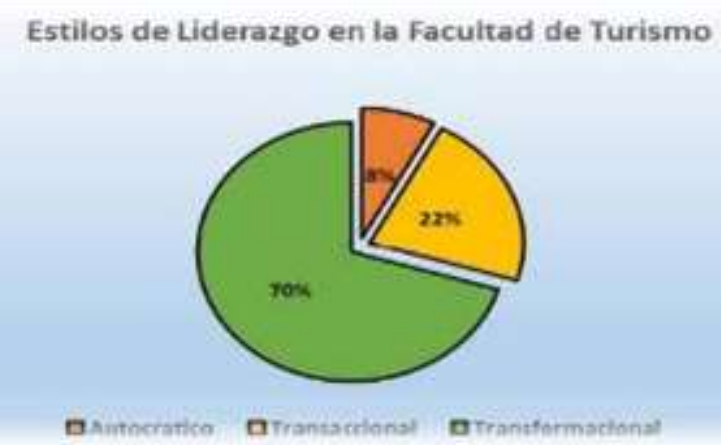

Figura 3. Estilos de Liderazgo en la Facultad de Turismo

Fuente: Elaboración propia

Desde la perspectiva de los directivos, las habilidades que se consideran con alto grado de positividad son: la perspectiva estratégica; capacidad para motivar a otros; ayudar al desarrollo de otros; asumir la responsabilidad, perseverancia; compromiso e implicación; honestidad; capacidad de servir a los demás; respeto. Se considera que no están totalmente óptimas: la comunicación; adquisición de experiencia; búsqueda de resultados; innovar y reinventarse; escuchar; persuadir; fortalecer relaciones sociales; delegar trabajo; aptarse al cambio; manejar la crisis; iniciativa y pasión por el trabajo; humildad para reconocer errores propios; detectar oportunidades; organizar; uso de pensamiento crítico y capacidad analizar y resolver. 


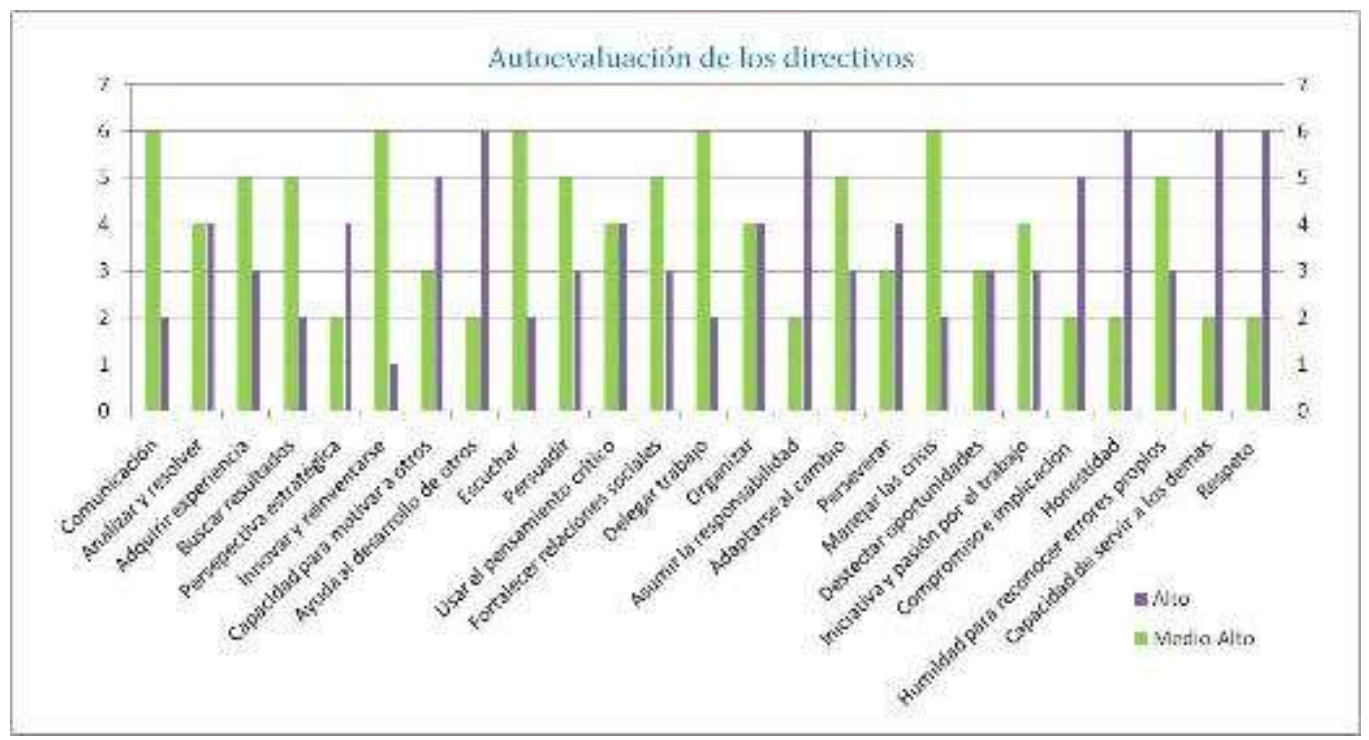

Figura 4. Autoevaluación de los directivos

Fuente: Elaboración propia

Desde la percepción de los profesores se consideran altamente positivas las habilidades: honestidad; asumir la responsabilidad y buscar resultados. Se consideraron que no están totalmente óptimas las habilidades: comunicación; capacidad de analizar y resolver; adquirir experiencia; perspectiva estratégica; innovar y reinventarse; capacidad para motivar a otros; ayudar al desarrollo de otros; escuchar; persuadir; uso de pensamiento crítico; fortalecer relaciones sociales; respeto; capacidad de servir a los demás; humildad para reconocer errores propios; compromiso e implicación; iniciativa y pasión por el trabajo; detectar oportunidades; manejar la crisis; perseverar; adaptarse al cambio; organizar y delegar trabajo.

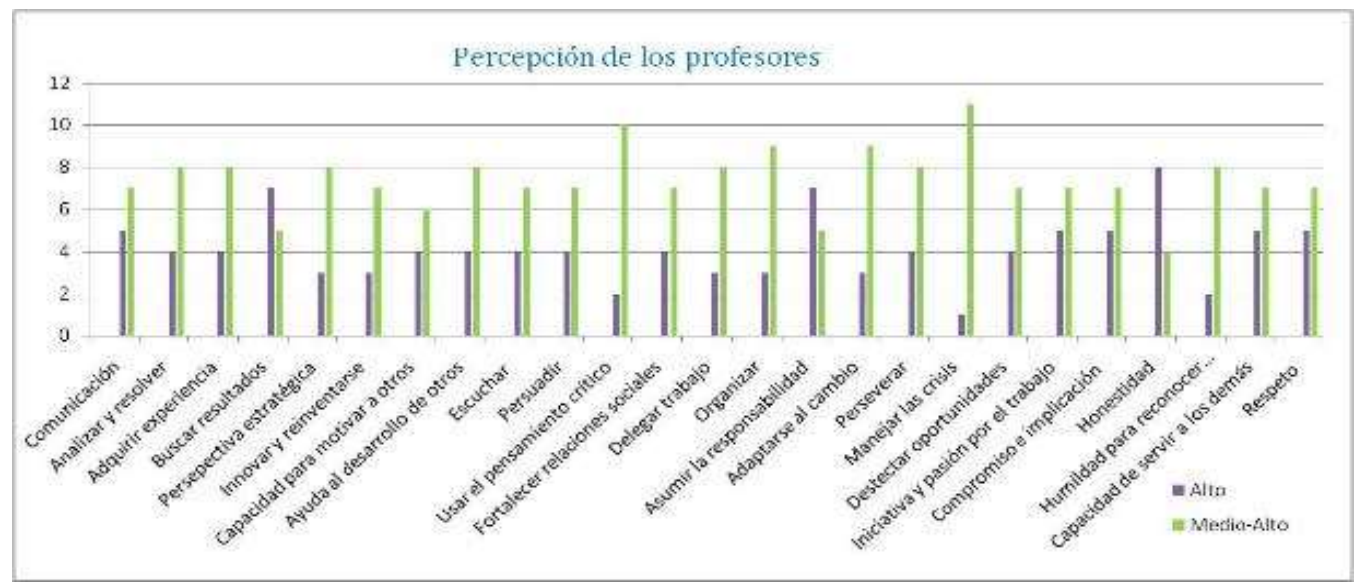

Figura 5. Percepción de los profesores

Fuente: Elaboración propia 
Estas dos perspectivas coinciden en que no está totalmente logradas las habilidades: comunicación; adquisición de experiencia; innovar y reinventarse; escuchar; persuadir; fortalecer relaciones sociales; delegar trabajo; adaptarse al cambio; manejar la crisis; iniciativa y pasión por el trabajo; humildad para reconocer errores propios. Las habilidades óptimamente logradas son: la honestidad y asumir responsabilidades.

Existe una diferencia de criterios en la evaluación de las habilidades: perspectiva estratégica; capacidad para motivar a otros; ayudar al desarrollo de otros; perseverar; compromiso e implicación; capacidad de servir a los demás y respeto; pues los directivos la calificaron como óptimas, mientras que los profesores consideran que no están totalmente logradas. Sucede lo contrario con la habilidad búsqueda de resultados, la cual fue catalogada como óptima por los profesores, mientras que los directivos consideran que no la tienen totalmente lograda.

Se tiene como particularidad en los resultados que las habilidades: detectar oportunidades; organizar; uso de pensamiento crítico; analizar y resolver fueron evaluadas por la mitad de los directivos como altamente positivas y la otra mitad consideró que se encontraba en un nivel medioalto. Según los resultados, los profesores corroboran que se encuentran en un nivel medio-alto.

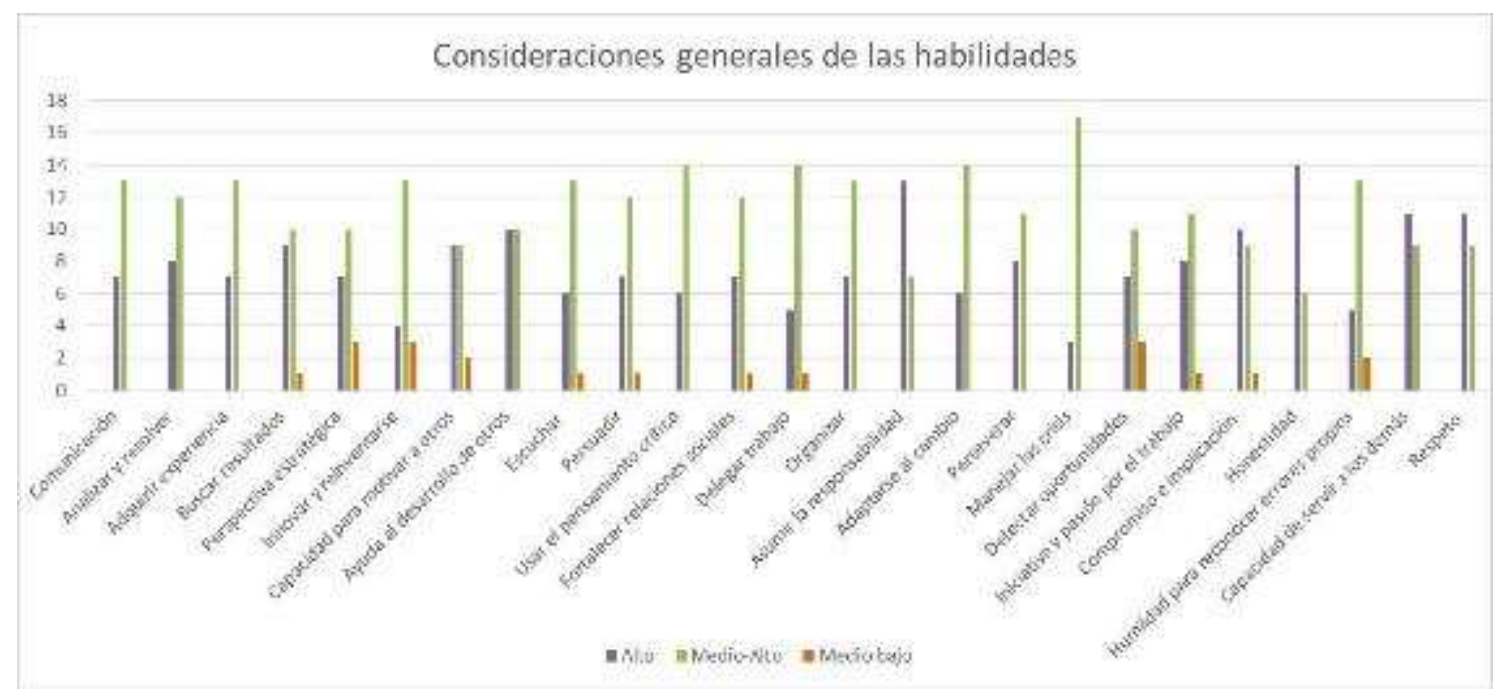

Figura 6. Evaluación de las habilidades.

Fuente: Elaboración propia

Desde la perspectiva de los estudiantes tenemos en un alto nivel en las habilidades: comunicación; escuchar; capacidad de servir a los demás; humildad para reconocer errores; fortalecer relaciones; 
capacidad de organizar y respetar. En un nivel medio-alto se sitúan: persuasión, perseverancia; iniciativa y pasión por el trabajo y honestidad. En un nivel medio catalogaron a: capacidad de motivar y ayudar al desarrollo de otros.

Luego del análisis de los datos se puede concluir que tienen un alto nivel las habilidades de honestidad y asumir responsabilidades. El resto se encuentra en un nivel medio-alto, por lo que se puede inferir que existen elementos que está influyendo negativamente en su logro total. Teniendo en cuenta las interpretaciones de las consideraciones de los profesores y estudiantes, las habilidades que se encuentran en una línea más débil con respecto a las demás son: manejo de las crisis, perseverancia, adaptación al cambio, delegar trabajo, uso del pensamiento crítico, persuadir, ayudar al desarrollo de otros.

\section{Resultados de la aplicación de la matriz de base estructural}

Con el fin de destacar las variables más influyentes en los actores que tributan a la Facultad de Turismo de la Universidad de La Habana desde el entorno o internamente, se hizo necesario realizar el análisis estructural. Para ello se definieron tres etapas que permitieron un perfecto análisis de dichas variables:

1- inventario de variables / factores: Para el desarrollo de esta etapa se recopiló una serie de variables, definiendo como resultado final 16 a analizar:

\begin{tabular}{|c|}
\hline Clima social/ambiente V1 \\
Movilización/motivación/convivialidad V2 \\
Interés del puesto de trabajo V3 \\
Cualificación/formación/reclutamiento V4 \\
Integración al inicio V5 \\
Condiciones de trabajo V6 \\
Rol de los administradores V7 \\
Valores culturales V8 \\
Medios-comunicación V9 \\
Calidad del servicio V10 \\
Proyecto de institución V11 \\
Política de imagen de empresa V12
\end{tabular}




\section{Integración de nuevos procesos V13 \\ Rentabilidad de los capitales \\ comprometidos V14 \\ Cambios de la tecnología V15 \\ Estrategia de los competidores V16}

2- Descripción de las relaciones entre variables en la Matriz de Base Estructural. Permite identificar las variables que más influyen sobre otras, lo que contribuye a la determinación de variables esenciales.

3- Identificación de variables esenciales: se identificaron como variables esenciales V1, V2, V4, V6, V7 y V10.

Para contrastar estos resultados se utilizó el método de grupo de especialistas con el cual se analizó la influencia que presentan estas 16 variables sobre las habilidades directivas de los cuadros de la Facultad. Llegando a la conclusión que en comparación con el análisis estructural que como esenciales resultaron las variables: V1, V2, V4, V6, V7, V8 y V10 con este método quedaron las variables $\mathrm{V} 1, \mathrm{~V} 2$, V4 y V7.

Como resultado de los análisis se proponen las siguientes estrategias:

Estrategia 1: Aumentar la información a los directivos de sobre las habilidades necesarias para el desempeño de su cargo.

Estrategia 2: Disminuir los factores que influyen negativamente en la mejora de las habilidades directivas.

Estrategia 3: Programar actividades para la formación de habilidades directivas dentro del sistema de capacitación de los directivos.

Estrategia 4: Instituir mecanismos que garanticen la efectividad del proceso de formación de habilidades directivas.

Estrategia 5. Implementar programa de capacitación para fomentar las habilidades de comunicación en los líderes. (Para habilidades relacionadas con la comunicación, capacidad para motivar a otros, escuchar, persuadir, honestidad). 


\section{CONCLUSIONES}

A partir de la aplicación del análisis de los métodos empleados para el estudio de las habilidades directivas, y el diseño estratégico propuesto para la empresa se puede arribar a las siguientes conclusiones:

- El estudio realizado en la Facultad de Turismo evidencia que existe un desarrollo positivo de las habilidades directivas en torno al liderazgo de sus dirigentes.

- Las habilidades de liderazgo mejor logradas en los directivos de la facultad son la honestidad y la asunción de la responsabilidad, mientras que las más débiles fueron el manejo de crisis, la adaptación al cambio, el uso del pensamiento crítico y la influencia y persuasión.

- El estilo de líder predominante en la facultad es el Transformacional, como un aspecto favorable que se debe generalizar en todos los directivos.

- Mediante el análisis estructural se determinó que las variables factores que más influyen son: clima social/ambiente, movilización/motivación/convivialidad, interés del puesto de trabajo, cualificación/formación/reclutamiento y rol de los administradores.

\section{REFERENCIAS BIBLIOGRÁFICAS}

Ascón, J.E. (2017) La dirección estratégica en empresas del sector de los servicios. Disponible en: www.gestiopolis.com Consultado el 12.12.17

Astigarraga, E. (2016). Prospectiva Estratégica: orígenes, conceptos claves e introducción a su práctica. Revista Centroamericana de Administración Publica, 71.

Bayón, F. (2015). Obtenido de Cómo ha cambiado el concepto de liderazgo: http://www.eoi.es/blogs/fernandobayon/2015/04/24/como-ha-cambiado-el-concepto-deliderazgo/

Berger ,G., 1959, "L'attitude prospective » in Berger (Gaston), Bourbon-Busset (Jacques, de), Massé (Pierre), 2007, De la Prospective. Textes fondamentaux de la prospective française (1955-1966), op. cit. 
Chamorro, D. (2005). Factores determinantes del estilo de liderazgo del director-a. Madrid.

Codina, A. (2001). 10 Habilidades Directivas. ¿Por qué? ¿Para qué? ¿Cómo?. Consultado el 30 de octubre de 2017, de http://www.degerencia.com/acodina

Codina, A. (2012). ¿Qué hacen los directivos y qué habilidades necesitan para un trabajo efectivo?. Consultado el 30 de octubre de 2017, de http://www.degerencia.com/acodina

Cristancho, B.; Rojas, A. M., Ensayo "El desarrollo eficiente de las habilidades gerenciales como herramienta de gestión". Universidad Militar Nueva Granada. Facultad de Ciencias Económicas. Especialización en alta gerencia. Bogotá. Junio, 2012.

Godet, M., ¿ Por qué es necesario analizar y construir el futuro? Donud. 2016

Godet, M. (2012). La Caja de Herramientas de la Prospectiva Estratégica.

Gómez, R. (2008). El liderazgo empresarial para la innovación tecnológica en las micro, pequeñas y medianas empresas. México.

Guerra, Y. Estrategias para la gerencia de restaurantes. Universidad de La Habana. Documento Word. 2009.

Leadership-Central. (2016) Obtenido de Leadership Theories: http://www.leadershipcentral.com/leadership-theories.html

Menguzzato, M., \& Renau, J. (1993). La dirección estrategica de la empresa: Un enfoque innovador del management.

Madrigal, B. E., Habilidades Directivas (segunda edición). Editorial The McGraw-Hill Companies, Inc. México. 2009

Miklos, T., \& Tello, M. (2007). Planeacion Prospectiva: Una estrategia para el diseño del futuro. 
Ronda, G.A. Dirección Estratégica integrada: Conceptualización en las condiciones de Cuba.Editorial Felix Varela. 2016.

Ronda, G. A. y Marcané J.A., Ciencias de la Información Vol. 35, No. 1, abril, 2004.

Ronda, G.A. Dirección estratégica, constructo y dimensiones. Ediciones Futuro. La Habana. 2015.

Ryo. (2015). Skeptikai. Obtenido de The Evolution of Leadership: http://skeptikai.com/2015/12/08/the-evolution-of-leadership/

Sutevski,D. (2009). Managerial skills- 3 Types of skills each manager will need.

Consultado el 30 de octubre de 2017 de

http://www.entrepreneurshipinabox.com/202/managerial-skills/

Whetten, D. A.; Cameron, K. S., Developing Management Skills (eighth edition). Prentice Hall. United States of America. 2011 